\title{
Una visión global del e.Learning y su e.Market
}

Guillermo Vázquez, Juan Lago, David Riley

Fundacion IAVANTE, Consejería de Salud de Andalucía

Las Tecnologías de la Información y de la Comunicación y su aplicación en la formación y entrenamiento de los profesionales, mediante el eLearning, supone una de las grandes revoluciones que caracterizan a la Sociedad del Conocimiento. Sin embargo solo una estrategia global que encare todas sus facetas simultáneamente puede acompasar el desarrollo tecnológico y su implementación efectiva. En este artículo los autores subrayan en primer lugar la importancia del liderazgo en la fase actual de implantación y aceptación del eLearning, y los punto claves sobre los que se debe de sustentar. En segundo lugar explican las características de las acciones formativas online, y su engarce en un Campus Virtual. En tercer lugar se explican las características tecnológicas del elearning. Finalmente recalcan la necesidad de un eMarket para asegurar que lo altos costes de una nueva metodología, son sostenibles.

\footnotetext{
Correspondencia:

G. Vázquez

Director de I+D+i Fundacion IAVANTE

Parque Tecnológico de Ciencias de la Salud

Avda de la Ciencia $\mathrm{s} / \mathrm{n}$

18.100 Armilla; Granada

Email: guillermo.vazquez@iavante.es

J. Lago: juan.lago@iavante.es

D. Riley: david.riley@iavante.es
}

\section{e.LEARNING COMO INNOVACIÓN DISRUPTIVA DE LOS MODELOS EDUCATIVOS HISTÓRICOS}

La educación a lo largo de los siglos ha quedado enmarcada por la transmisión oral del conocimiento, su compilación como conocimiento codificado en los libros, su difusión en escuelas y universidades y su adquisición en las primeras décadas de la vida. Los títulos universitarios que a lo largo de los siglos se ha ido otorgando de manera secuencial, bachilleres, licenciados, doctores y actualmente "master" reflejan la complejidad creciente de los conocimientos y habilidades a adquirir. Sin embargo en la sociedad actual, es decir la denominada "Sociedad del Conocimiento", existen nuevos paradigmas. En primer lugar el conocimiento y su aplicación en la práctica diaria avanza muy rápidamente, lo que repercute en los modos de trabajar y de producir, lo que a su vez conlleva que los ciudadanos tengan que reciclar sus conocimientos y habilidades periódicamente para mantener sus competencias y "empleabilidad" (denominación en uso en la Unión Europea para subrayar la necesidad de que la formación promueva la accesibilidad al trabajo) ${ }^{1}$. Esta situación presupone que el aprendizaje va mas 
allá del periodo universitario, abarcando toda la vida útil, conociéndose este nuevo paradigma con las palabras anglosajonas "Lifelong learning". En segundo lugar, el incremento de la población en todos los países desarrollados y en vías de desarrollo, hace prever que las estructuras educativas actuales queden sobrepasadas físicamente, debiéndose buscar nuevas alternativas a las mismas. Finalmente el desarrollo de las Tecnologías de la Información y de la Comunicación, (TIC), han producido una revolución en el campo de la educación de pregrado y postgrado, al permitir romper los límites de la enseñanza clásica reseñados anteriormente; el alumno o usuario puede recibir la formacion adecuada a sus necesidades en cualquier lugar geográfico en que se encuentren, es decir los límites físicos y temporales desaparecen ${ }^{2}$; se promueven nuevos medios de relación interactiva profesor alumno; el libro escrito, indistintamente que se siga utilizando, tiene su contrapartida en los materiales interactivos que se posibilitan con las $\mathrm{TICs}^{3}$, combinado con el acceso a toda la información necesaria a través de las librerías virtuales. Esta nueva herramienta se denomina "e.Learning", aunque también se conoce con otras denominaciones, tales como formacion Online, o Web Based Learning; nosotros utilizaremos en este articulo e.Learning por ser la mas extendida y aceptada actualmente ${ }^{4}$. Este tipo de formacion se recibe fuera del aula clásica, a través de Internet o intranet, y normalmente se apoya en conjunto de prestaciones virtuales administrativas y de apoyo al alumno que se denominan "Campus Virtual". A pesar de todos los avances tecnológicos, existe un retraso en su implantación y utilización. Solo una aproximación global al e.Learning puede promover su empleo y difusión y anular dicho retraso.

\section{EL LIDERAZGO EN LA FORMACION e.LEARNING}

Las TICS son una decisión estratégica en todos los campos incluyendo la educación y por tanto requieren un liderazgo; este liderazgo es necesario dado que las TICs aplicadas en e.Learning son una innovación disruptiva, es decir una tecnología que sustituye las previas, y abocada a cambiar todo el escenario docente ${ }^{5}$. No es aventurado avanzar que posiblemente se convierta en el modelo predominante en un futuro ${ }^{6}$. Las TICs aplicadas a la formacion y entrenamiento de los profesionales de la sanidad, requiere un liderazgo fuerte que entienda la revolución que suponen estas tecnologías, las apoye y les dé continuidad. Esta función de liderazgo se debe de reforzar los siguientes puntos.

a. Identificar el nivel de digitalización de los profesionales de su institución, promover su formacion permanente en TICs y eLearning, potenciar su participación activa en la implantación del e.Learning y fomentar su participación en reuniones y actividades científicas relacionadas con las TICs.

b. Promover la formacion de tutores y profesores en eLearning, para convertirlos en agentes multiplicadores del cambio de paradigma educativo

c. Entender que la formacion y entrenamiento informal pueden jugar un papel importante para romper las barreras no solo tecnológicas sino también psicológicas de las plantillas para adoptar y adaptarse a las nuevas tecnologías,

d. Conocer que la innovación conlleva una serie de normas, cuya comprensión mejora las estrategias a seleccionar. La curva de innovadores descrita por Rogers ${ }^{7}$ hace varias décadas pero aún vigente, cuantifica las fases por las que pasa una plantilla para la adopción de una innovación; esta curva permite una aproximación a las estrategias a implantar según la situación en la que nos encontremos en la curva. Cada etapa descrita requiere la consolidación de la previa, y cada etapa requiere entender el perfil profesional que la caracteriza. La masa crítica para promover el cambio se alcanza cuando el grupo de innovadores llega al $15 \%$.

e. Conocer las opciones de harward y plataformas tecnologías sobre las que construir los cursos, y el coste económico global que conlleva, tanto su implantación como sus mantenimiento. Para esto deberá de asesorarse adecuadamente, para decidir la opción mas provechosa para su institución.

d. Identificar que el e.Learning y las infraestructuras que lo soportan, tanto tecnológicas como funcionales, requieren puestos de trabajo con perfiles profesionales nuevos (ingenierías, psicólogos, pedagogos, o diseñadores)

f. Introducir paulatinamente cambios en el Currículo Universitario, o de postgrado (Carrera Profesional), potenciando una visión mas personalizada del mismo, empotrado ("Embeded, según la terminología anglosajona) en el trabajo, y adaptado a las necesidades y disponibilidades del estudiante / usuario.

\section{LOS CURSOS}

La formacion e.Learning al igual que en otras modalidades, debe de diseñarse para activar el cono- 
cimiento previo, y complementarlo con nuevas adquisiciones, es decir potenciando la metodología constructivista. La formacion e.Learning debe de buscar la multi-dimensionalidad, el trabajo en equipo, y su desarrollo bien contextualizado ${ }^{7}$. La formación mediante e.Learning debe de caracterizarse por:

a. Tener un conocimiento codificado, fácilmente navegable, acompañado de comentarios y notas aclaratorias, junto con una gran interactividad, y "links" con otros Web-sites.

b. Microsimulaciones, es decir video juegos, en los que se trabaja no solo los contenidos multimedia sino que también permiten adquirir habilidades psicomotoras mediante interfases adecuadas.

c. Aula virtual, que sustituye o se complementa con las presenciales, cumpliendo las mismas funciones que esta última, mediante diferentes medios audiovisuales.

d. Comunidades virtuales, para potenciar el trabajo colaborativo.

e. Comunicación profesor/alumno-s, mediante diferentes herramientas

f. Librería virtual, y accesibilidad a otra fuentes de información de calidad para construir el conocimiento.

g. Soporte administrativo para matricularse, y cumplimentar todos los trámites necesarios.

h. e.Repositorio donde se puedan encontrar los recursos formativos y seleccionar aquellos que mas se amoldan a las necesidades personales, junto un e.Portfolio para objetivar las competencias adquiridas y su aplicación.

La construcción de estos cursos sobrepasa la preparación y disponibilidad de tiempo de los profesores, cuya área de excelencia es casi siempre clínica o de investigación experimental, por tanto es necesario tener un equipo de apoyo. Nuevas profesiones y nuevos puestos de trabajo se requieren para afrontar este reto. El coste de estos diseños, y de su infraestructura supera ampliamente la estructura clásica de las Universidades, o de cualquier comisión de docencia hospitalaria y centros de familia.

\section{LOS ELEMENTOS ESTRUCTURALES DEL E.LEARNING}

Tal como hemos venido insistiendo, la generalización de Internet y de las redes de Intranet, ha revolucionado con el e.Learning el campo de la formacion y entrenamiento, así como otros campos de la medicina como es la Telemedicina, o la relación con el ciudadano / enfermo como es el eSalud.

Todos estos campos descansan en infraestructuras tecnológicas que van más allá del ordenador personal ${ }^{4-8}$. Estas tecnologías permiten una extraordinaria movilidad del usuario, una gran interconectividad y manejar la información empotrada en su actividad. Abarcan toda la infraestructura de servidores (servidores Web, plataformas e.Learning, servidores de contenidos, servidores de Base de Datos, etc), las redes de comunicación, los ordenadores de sobremesa, portátiles y de mano "PDA" (Personal Digital Assistant) dotadas con todo tipo de prestaciones, los reproductores de audio/vídeo cada vez más reducidos en tamaño pero más potentes en prestaciones, y finalmente la telefonía móvil de tercera generación destacando las posibilidades de conectividad a Internet desde cualquier punto incluso en movimiento y las video-llamadas.

Los cursos y su material pueden incluir contenidos en distintos formatos como texto, imágenes, audio, vídeo, animaciones, etc. apoyados en herramientas colaborativas asíncronas como foros, wikis, blogs y herramientas síncronas como videoconferencias, mensajería instantánea tanto uno a uno como en grupo.

Paralelamente un conjunto de elementos de soporte administrativo para el alumno / profesional, y otros de soporte de diseños, y manejo de recursos para los profesores / tutores. En la fundacion IAVANTE, disponemos de una solución propia mediante la Web-site SIGESxComp (Sistema Integral de Gestión por Competencias) que engloba todas estas funciones en módulos, Formacion, Competencias, Plan de Desarrollo Profesional, Logística y Gestión del Conocimiento ${ }^{9}$.

Los diversos apartados que constituyen el e.Learning se manejan a través de dos plataformas de software complementarias y actualmente con tendencia convergente:

a.-Learning Management System (LMS): plataforma de formación orientada inicialmente hacia la planificación, entrega y gestión de cursos. Entre otras funcionalidades incluye la creación de cursos, alta de usuarios de distintos perfiles (gestores, tutores, alumnos), matriculaciones, aula virtual y parte o todas las herramientas colaborativas comentadas anteriormente. Permite realizar un seguimiento de los alumnos tanto de actividad como de evaluación de conocimientos adquiridos permitiendo extraer informes. 
b.-Learning Content Management System (LCMS): plataforma centrada en los contenidos propiamente dichos. Más orientada a autores de contenidos, diseñadores instruccionales y expertos de un determinado área del conocimiento.

Estas plataformas pueden ser propietarias o bien de código abierto (Open Source), debiendo decidir las instituciones aquella opción que mas le conviene y se ajusta a su necesidades y disponibilidades económicas.

Esta breve exposición pone de relieve la necesidad de que las instituciones tengan un equipo de apoyo al elearning, y que los profesores tengan un nivel de digitalización adecuado. Otra característica que fácilmente se deduce es el alto coste del elearning.

\section{e.LEARNING Y e.MARKET}

Todo lo expuesto hasta aquí apunta a unas necesidades presupuestarias elevadas, y dentro de ellas uno de los capítulos a tener en cuenta es el diseño de los cursos de e.Learning y sus contenidos. Estos contenidos, por su elevado costo deben de rentabilizarse a través de su utilización en diversas plataformas y organizaciones. Sin embargo esta rentabilización, en la cual subyace las posibilidades de crear un e.Market de contenidos y cursos de elearning, requiere.de un conjunto de características que enumeramos brevemente.

a.-En primer lugar se requiere la interoperabilidad entre las plataformas, de manera que el curso pueda utilizase en diversos ambientes sin cambios y de forma amigable. A su vez sus contenidos deben ser reutilizables para implementar el mismo curso $\mathrm{u}$ otros, lo que fuerza a estructurar muy bien los objetos de aprendizaje. El SCORM (Sharable Content Object Referente Model) es un conjunto de estándares y especificaciones para la construcción de las acciones de e.Learning que permitan su interoperabilidad y reutilización. Otros capítulos de esta monografía desarrollan este concepto con más extensión.

b.-Además los cursos deben de estar diseñados por diferentes partes independientes entre sí, denominadas "Learning Objects" que permiten ser reutilizadas en nuevas acciones formativas (ej. Una simulación de funcionamiento cardiaco puede servir para explicar fisiología cardiovascular, la insuficiencia cardiaca, o el cateterismo de sus cámaras).

c.-De manera similar al concepto de biblioteca virtual donde se pueden encontrar todas las publicaciones científicas, deben de existir eRepositorios donde la persona interesada pueda encontrar los cursos que necesita para su entrenamiento permanente, y los profesores y los equipos de diseño "Learning Objects" para sus acciones formativas.

d.-Finalmente es necesario regular los derechos de autor, y todos aquellos concepto de privacidad necesarios.

e.-También es necesario que las normativas de Créditos de e.Learning estén bien definidas cumpliendo con las especificaciones de la Declaración de Bolonia de la Unión Europea, así como el Suplemento al Título Recomendado en el Espacio Europeo del Conocimiento, donde se especifiquen las competencias adquiridas y el modo en que se han obtenido ${ }^{1}$. Esto dos último conceptos buscan la movilidad de los profesionales en el espacio de la Unión Europea, y son también un aliciente mas para la creación de alianzas y mercados para el e.Learning.

Por tanto cabe esperar que los próximos años se vaya definiendo claramente un modelo de negocio que permita al e.Learning despegar, y por tanto implementar todas sus potencialidades de cambio disruptivo en la educación.

\section{BIBLIOGRAFÍA}

1. European Comission. Education and Training. Bologna Process http://ec.europa.eu/education/index_en.html

2. J Ruiz, Mj Mintzer, Rc Leipzig. The Impact of e.Learning in medical education. Acad Med 2006; 86:207-212

3. J Wiecha, R Grambling, P Joachim. Collaborative online learning: a new approach to distance CME. Acad Med.2002; 77:928-929

4. J Mckimm, C Jollie, P Cantillon. ABC of learning and teaching Web Based Learning. BMJ 2003; 326:870-873

5. S Chodorow. Educators must take the electronic revolution seriously. Acad Med 1996; 71: 221-26

6. JP Ward, J Gordon, MJ Field, HP Lehmann. Communication and Information Technology in medical education. Lancet 2001; 357:792-796

7. T. Grunwald, Ch Corsbie-Massay. Guidelines for cognitive Efficient Multimedia Learning Tolls: Educational Strategies, Cognitive Load, and Interface Design. Acad Med 2006; 81:213-223

8. T. Greenhalgh. Computer assisted learning in undergraduate medical education. BMJ 2001; 322:40-44

9. IAVANTE Fundación. SIGES X COM. http://www.iavantefundacion.com/portal3d/default.htm 